\title{
Educação interprofissional na graduação em saúde: aspectos avaliativos da implantação na Faculdade de Medicina de Marília (Famema)
}

\section{Interprofessional education in health graduation: evaluation aspects of its implementation in Marilia Medical School (Famema)}

\author{
Rinaldo Henrique Aguilar da Silva ${ }^{1}$
}

\begin{abstract}
RESUMO
O objetivo deste trabalho é realizar uma reflexão sobre a implantação da educação interprofissional em saúde da Famema, destacando as principais fragilidades e fortalezas relatadas, por professores e estudantes, durante o processo de avaliação. A educação interprofissional vem sendo utilizada como estratégia de ensino em vários países visando à formação de profissionais mais críticos, reflexivos, capazes de trabalhar em equipe e de aprenderem juntos com as outras profissões. Foi possível verificar que na Famema as fragilidades e fortalezas apresentadas referiram-se aos seguintes aspectos: a) entendimento do que é educação interprofissional; b) o trabalho em equipe na atenção primária em saúde; c) os conteúdos das especialidades e o ganho de conhecimento cognitivo; d) a avaliação como momento de aprendizagem. Conclui-se que apesar dos grandes desafios ainda existentes, este modelo de educação interprofissional na Famema está em consonância com a imagem objetivo proposta.

Palavras-chave: educação interprofissional; avaliação; graduação em saúde.
\end{abstract}

1 Pós-doutorando em Ensino Superior em Saúde pela Universidade Federal de São Paulo (UNIFESP) - Mestre e Doutor em Genética e Evolução - Especialista em Administração Universitária e Gestão Acadêmica - Docente da Faculdade de Medicina de Marília (Famema),São Paulo, Brasil.E-mail-aguilar@famema.br 


\begin{abstract}
This paper aims at thinking on the implementation of health interprofessional education program in Famema and pointing out the main weaknesses and strengths reported by teachers and students during the evaluation process. Interprofessional education has been used as a teaching strategy in several countries to educate more critically, reflexively the professionals who are able to work in a team and to learn together with other professions. It was possible to see that in Famema the presented weaknesses and strengths were related to the following aspects: a) understanding what is interprofessional education; b) team work in primary health care; c) the specialties' contents and broader cognitive knowledge; d) evaluation as a learning instance. One may conclude that in spite the challenges this interprofessional education pattern in Famema is according to the proposed objective.

Keywords: interprofessional education; evaluation; health graduation.
\end{abstract}

\title{
Introdução
}

Uma das questões que se coloca em relação à consolidação de práticas de saúde que compõe um modelo calcado nos princípios e diretrizes do Sistema Único de Saúde (SUS), é a problemática da força de trabalho, sendo que esta deve ser enfrentada tanto do ponto de vista das práticas como da formação de pessoal.

Aponta-se ao longo dos anos de implementação do SUS que a formação da força de trabalho em saúde proporcionada pelo aparelho formador não é adequada às necessidades de saúde da população brasileira nem às necessidades dos serviços (BRASIL, 1993; NOGUEIRA, 2002).

Assim, a formulação de novas propostas para a formação dos profissionais da área da saúde, na perspectiva crítica e interprofissional, ou seja, a formação crítico-reflexiva e colaborativa, na direção da constituição de sujeitos para a transformação social, consideram-se como uma das demandas para que se reorganizem as práticas de saúde na direção de um sistema ancorado nos princípios e diretrizes do SUS. No entanto, vários aspectos apresentam-se como motivos de uma crise na formação dos recursos humanos, gerados por determinantes externos e internos aos órgãos formadores.

No Brasil como em vários países no mundo, questiona-se sobre qual o papel do sistema educacional, incluindo o universitário, no processo de formação das pessoas na sociedade, bem como de sua articulação com setores específicos desta (SANTOS, 1999). 
A educação não comporta mais uma bagagem escolar baseada no volume de conteúdos e nas especificidades de cada profissão, sendo isso pouco operacional e nem mesmo adequado. O que se acumula no começo da vida deve ser constantemente atualizado e aprofundado, considerando que estamos vivendo um período no qual às mudanças ocorrem com grande velocidade. Além disto, é essencial que os profissionais possam trabalhar colaborativamente compartilhando ações e conhecimentos. Ressalta-se também que a educação, para dar resposta ao conjunto das suas missões, deve organizar-se em torno de quatro aprendizagens fundamentais, que serão ao longo da vida os quatro pilares do conhecimento: aprender a conhecer (ou aprender a aprender, adquirir os instrumentos de compreensão), aprender a fazer (para poder agir sobre o meio envolvente), aprender a viver juntos (a fim de participar e cooperar com os outros em todas as atividades humanas) e aprender a ser (via essencial que integra as três precedentes) (DELORS, 2000).

Há que se constituírem novos processos provocando e desencadeando discussões acerca da prática em saúde, de forma que essa seja revisitada, retomando sua finalidade, questionando o compromisso com os segmentos da sociedade e a articulação dessa prática com o processo de formação dos profissionais de saúde como possibilidade de formação de sujeitos.

Nessa perspectiva, há necessidade de se redirecionar a formação inicial, colocando em questão os modelos e valores a serem construídos na prática, para atender a esta reorganização, pautando-se pela ética nos processos de intervenção, enquanto direito e respeito ao ser humano, construindo e re-construindo os processos educacionais e competências profissionais, integrando a formação à realidade dos serviços da comunidade.

No entanto, a sociedade vive em constante movimento e tensão, tendo forças favoráveis e desfavoráveis a cada projeto implementado. Da mesma forma, a educação vive momentos nos quais seus projetos podem servir tanto para a reprodução dos processos na sociedade de forma acrítica, como para desenvolver uma formação crítica, reflexiva, comprometida e consciente para atuar a favor da emancipação das pessoas, da igualdade social e da qualidade de vida, formando-se cidadãos no seu processo de humanização.

Em vários países, experiências de educação interprofissional na graduação em saúde (HIND et al., 2003; MCNAIR et al., 2005; COOPER et al. 2005; GOELEN et al., 2006) apresentam mudanças consistentes no perfil dos profissionais formados. Estas possibilitam a discussão sobre a intencionalidade deste tipo de formação, apontando o desafio de se ter domínio de conhecimentos, habilidades e atitudes para atuar com competência, com qualidade formal e política e, ao mesmo tempo, responder às necessidades sociais. Abordam ainda questões ético-políticas que podem auxiliar na otimização dos recursos de saúde 
e desenvolver a consciência nos atos/escolhas praticados já que passam a ser discutidos criticamente em equipe.

Assim, considerando a concepção de saúde-doença na perspectiva da qualidade de vida, na reorganização do setor saúde, através de ações de promoção, prevenção e recuperação, há necessidade também de se promover transformações na formação profissional brasileira, sendo um desafio à construção de novas tecnologias para a intervenção. Estas mudanças devem buscar a articulação entre teoria e prática, buscar a integração ensino-serviço-comunidade, influenciar a instrumentalização dos novos profissionais que saibam e gostem de trabalhar em equipe.

Importante ressaltar que a prática não deve se reduzir a eventos empíricos ou ilustrações pontuais, mas deve exigir uma intencional e contínua postura de problematização do vivido, observado, discutido. Deve-se possibilitar que o estudante lide com a realidade e dela retire elementos que conferirão significado e direção às aprendizagens (BATISTA; BATISTA, 2008).

Minayo (1997) aponta que, em função da globalização, o mundo do trabalho é o setor em que as mudanças se fazem mais presentes. O avanço científico e tecnológico coloca, nesse momento histórico, o conhecimento como a força produtiva por excelência, exigindo uma (re)discussão dos processos de formação dos profissionais para que esses possam ser inseridos num mercado de trabalho em constante transformação.

Hoje não faz mais sentido pensar o conhecimento fora do mundo do trabalho, e os profissionais não trabalham sozinhos e nem tampouco somente com seus "iguais". Assim, a convivência e partilha de conhecimentos visa transformar o processo de trabalho numa relação estreita entre teoria e prática, num movimento dinâmico, a práxis.

Dessa forma, toda atividade, ao incorporar uma intencionalidade, ou seja, uma finalidade previamente elaborada pelo sujeito torna a ação consciente, relacionando de forma indivisível a teoria e a prática, a prática consciente, dinâmica, podendo ter a interferência dos seres humanos na transformação da realidade.

A finalidade de todo o processo está no entendimento do que subjaz às aparências na realidade, ou seja, a construção de uma leitura crítico-reflexiva da realidade, transformando o estudante/professor/profissional da saúde em sujeito, determinando seu processo de formação e ao mesmo tempo sendo determinado pelo mesmo.

Pensamos que tais elementos configuram a necessidade de melhor explorar e compreender os determinantes das tensões existentes nestes espaços, visando o estabelecimento de estratégias para a implantação de processos de educação interprofissional que contribuam para as mudanças requeridas. Corroborando com estas ideias, Motta et al. (2001) enfatizam a necessidade de estabelecer 
diálogos no sentido de aprofundar a compreensão dos contextos em que se dá a organização do trabalho e o papel das novas competências profissionais na sua dinâmica, com a finalidade de planejar estratégias, onde a educação aparece como uma importante mediadora que permite o "deslocamento de ações pedagógicas para o espaço de realização do trabalho." (MOTTA; BUSS; NUNES, 2001, p. 06), favorecendo articulações para a obtenção de impacto no sistema como um todo.

Ao enunciar esta concepção, busca-se recuperar a capacidade do ser humano, na qualidade de estudante, de ser sujeito do seu processo de formação, construindo-se a partir daí a possibilidade do mesmo ser ativo na sua produção, articulado às necessidades sociais, vivenciando um projeto constituído interprofissionalmente, de forma que a sua prática tenha compromisso e responsabilidade com o usuário do sistema de saúde e que possa impulsionar processos de mudanças na realidade onde se situa.

\section{Objetivo}

O objetivo deste trabalho é realizar uma reflexão da implantação da educação interprofissional da Faculdade de Medicina de Marília, destacando as principais fragilidades e fortalezas relatadas, por professores e estudantes, durante o processo de avaliação.

\section{Método}

\section{Natureza do estudo}

Tratou-se de uma análise de pesquisa avaliativa, o que segundo Contrandriopoulos et al. (1997), significa "examinar através de um procedimento científico as relações que existem entre os diferentes componentes de uma intervenção" e para tanto serão utilizadas as abordagens de pesquisa qualitativa que pretende aproximar-se de uma realidade com a finalidade de conhecê-la. (MINAYO; SANCHES, 1993) 


\section{Campo do estudo}

O campo é o recorte espacial que corresponde à abrangência, em termos empíricos, ao objeto de estudo (MINAYO, 2003), este compreende o contexto onde se desenvolve o objeto, sendo o espaço onde serão obtidos os dados empíricos sobre o mesmo.

Assim o campo determinado para este estudo foi onde se desenvolveram as atividades didáticas do ciclo de dois anos dos cursos de medicina e enfermagem da Famema. Para isso foram analisados os instrumentos de avaliação que foram aplicados a professores e estudantes ao final do segundo semestre do ano de 2008.

\section{Análise dos dados}

O método utilizado baseou-se na análise de conteúdo, modalidade temática, com ênfase na abordagem qualitativa de pesquisa social (BARDIN, 1979; MINAYO, 1992; GOMES, 2005). Esta técnica consiste em descobrir os "núcleos de sentido" que compõe a comunicação e cuja presença, ou frequência de aparição pode significar alguma coisa para o objetivo analítico escolhido.

\section{Resultados}

Foi realizada a leitura de uma amostra aleatória (50\%) dos instrumentos preenchidos por professores e estudantes ao final de cada unidade educacional, buscando uma compreensão global, na qual se privilegiou não apenas o conteúdo, como também sua lógica. Em seguida através de confrontos dos instrumentos foram identificados núcleos de sentido ou eixos que estruturavam os depoimentos, em torno dos quais se agrupavam características comuns (GOMES, 2005). Após identificadas as temáticas, em torno das quais os dados foram analisados e discutidos destacaram-se:

4.1 entendimento do que é educação interprofissional;

4.2 o trabalho em equipe na atenção primária em saúde;

4.3 os conteúdos das especialidades e o ganho de conhecimento cognitivo;

4.4 a avaliação como momento de aprendizagem. 


\subsection{Entendimento do que é educação interprofissional}

Na maioria dos instrumentos de avaliação os estudantes apresentam desconhecimento sobre o conceito de Educação interprofissional. Relacionaram o fato de estudarem juntos em grupos mistos como sendo uma consequência da metodologia problematizadora (Problem Beased Learning) adotada pela escola "[...] estamos juntos porque a Famema é uma escola PBL e aqui tudo é diferente"; "Achei estranho estudar junto com o curso de Medicina já que escolhi Enfermagem, mas acredito na nova metodologia já que faz muito tempo que a Famema forma assim e é reconhecida como uma ótima faculdade".

Para os professores encontramos uma duplicidade de entendimentos. Para alguns o conceito de educação interprofissional está relacionado ao mercado de trabalho "É preciso que os estudantes aprendam a trabalhar juntos, já que atualmente o mercado de trabalho exige esta competência"; "A concepção do SUS dentro da perspectiva do Programa de Saúde da Família pressupõe o trabalho colaborativo de médicos e enfermeiros". Para outros o trabalho em conjunto refere-se à necessidade de otimização de recursos "[...] e assim como o PBL trabalha com pequenos grupos, são necessários mais professores, por isso trabalhar em conjunto otimiza os recursos da escola e do serviço de saúde"; "Nós professores temos muito mais trabalho do que tínhamos anteriormente e por isso fica mais fácil trabalhar o que é comum dos dois cursos junto".

\subsection{O trabalho em equipe na atenção primária em saúde}

O trabalho em equipe foi apontado como sendo uma das fortalezas do processo, principalmente quando referido a atenção básica em saúde. Os alunos relatam " [...] trabalhando junto no Programa de Saúde da Família tenho a oportunidade de entender o papel de cada um no atendimento ao paciente"; "Aprendi que muitas das ações feitas no PSF podem ser compartilhadas tanto por médicos como por enfermeiros e como estudante de enfermagem me senti valorizada com isso". Para os professores parece não haver distinção entre os cursos na realização das tarefas "[...] e como as tarefas que são realizadas pelos estudantes estão bem descritas, sobretudo no que se refere ao cuidado individual à saúde, tanto estudantes de medicina como de enfermagem realizam de maneira satisfatória suas ações". Além disto, valorizaram o trabalho em conjunto "O trabalho em equipe fortalece vínculos, respeito mútuo e torna agradável a convivência 
entre estudantes e professores"; "[...] e assim trabalhando em equipe sinto que aprendo com os estudantes e eles aprendem comigo a cada dia".

\subsection{Os conteúdos das especialidades e o ganho de conhecimento cognitivo}

Entre as temáticas levantadas, esta apresentou-se como sendo a de maior fragilidade. Para os professores parece haver receio sobre o conteúdo das disciplinas e a capacidade de aquisição do conhecimento cognitivo, "[...] e portanto, acredito ser difícil que estudantes de enfermagem acompanhem os conteúdos de algumas disciplinas dentro da complexidade que deveria ser exigida para os estudantes de medicina"; "Tenho a impressão de nivelar ou por cima, ou por baixo, quando falo de conteúdos. Se por um lado acredito haver a necessidade de exigir mais para estudantes de medicina, por outro, tenho receio de que os estudantes de enfermagem não consigam acompanhar". Os estudantes relatam esta mesma impressão "[...] e sendo aluno da medicina tenho medo que estejam nivelando por baixo para que todos possam acompanhar"; "Nós alunos da enfermagem as vezes temos a impressão de que alguns professores acham que não somos capazes de acompanhar os conteúdos. Outro dia, em uma conferência, o professor só se referia aos estudantes de medicina e a todo momento perguntava se os alunos da enfermagem estavam conseguindo acompanhar".

\subsection{A avaliação como momento de aprendizagem}

A mudança do paradigma de formação exige também a mudança do processo avaliativo, pois demanda intenso envolvimento e entendimento do que é uma avaliação de caráter formativo. Os professores relataram a avaliação como um momento importante no diagnóstico e melhoria dos processos. Os professores relatam que "A avaliação é motivadora, pois sendo processual, possibilita a intervenção no momento em que o estudante mais precisa de ajuda"; "[...] sendo ela referenciada em critérios que levam em conta as habilidades e atitudes do estudante no campo de prática, este tem a possibilidade de desenvolver suas competências ao longo do curso de maneira mais acompanhada". Já os estudantes apontam tanto fortalezas no processo "[...] e é um momento de crescimento pessoal, porque é o momento que eu tenho para me avaliar"; 
"É uma oportunidade de fazer uma autoavaliação e melhorar o meu potencial" como aspectos de fragilidade que se referem mais à postura do professor no processo "O professor às vezes já conhece o estudante e leva isso em consideração, a empatia, para favorecer o estudante"; "O professor tem que desenvolver a capacidade de se adaptar ao processo e manter uma postura firme no momento da avaliação. Saber assumir sua posição de avaliador para não ficar baseado na empatia e afinidade pessoal".

\section{Discussão}

A Faculdade de Medicina de Marília, em seus Cursos de Enfermagem e Medicina, implementou o currículo fundamentado em Competência Profissional, centrado no estudante de acordo com os princípios da metodologia ativa de aprendizagem e educação interprofissional. Compõe um ciclo de dois anos comuns, que buscam desenvolver recursos nas áreas do cuidado individual, cuidado coletivo e de gestão dos serviços de saúde, na lógica da vigilância em saúde, com ênfase na atenção primária. Propõe que se alcance a competência, através da realização de determinadas tarefas, lançando mão de recursos, os quais serão desenvolvidos em cenários diversos (TSUJI ; SILVA, 2006).

As duas séries iniciais onde estudantes de medicina e enfermagem estudam, trabalham e aprendem juntos, estão formadas pela Unidade Educacional Sistematizada (UES), Unidade de Pratica Profissional (UPP) e Unidade Educacional Eletiva. Mantém os pressupostos curriculares da formação por competências profissionais e interprofissional. Existe também ligado à UPP, o Laboratório de Práticas Profissionais (LPP).(FIG. 1)

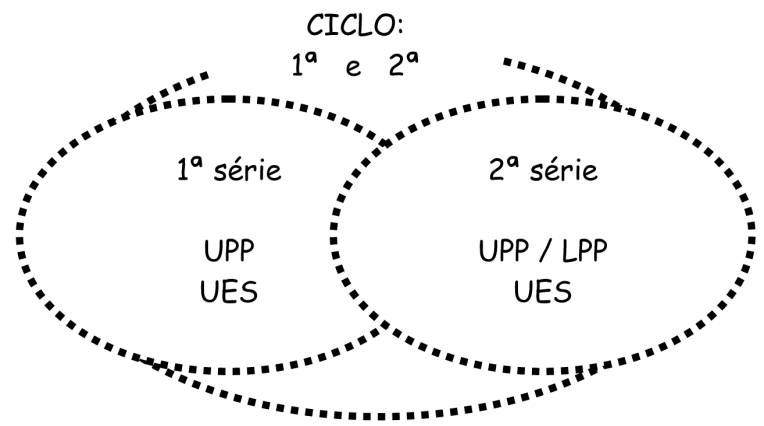

FIGURA 1 - REPRESENTAÇÃO ESQUEMÁTICADAARTICULAÇÃO ENTREAS UNIDADES EDUCACIONAIS 
O LPP é um momento sistematizado da aprendizagem, no qual as atividades são previamente estruturadas pelos docentes na forma de situações simuladas. Nos diferentes cenários de ensino/aprendizagem, utilizados para seu desenvolvimento, as atividades são realizadas em pequenos grupos e os estudantes são estimulados a problematizar as situações e desafiados a descobrir e conhecer os caminhos que lhes permitam aprender a aprender e construir sua formação profissional.

Em um esforço de articulação da UPP/LPP e UES são propostas tarefas que são realizadas no cenário da prática e que, também, são discutidas na UES proporcionando reflexão teórica que subsidiem a UPP/LPP.

Cada um dos cenários trabalha com predomínio de alguns recursos específicos, assim a UPP/LPP (espaços privilegiados da prática) trabalha com ênfase nos recursos psicomotores e afetivo e a UES com o recurso cognitivo.

As tarefas descritas estão organizadas em três núcleos de conhecimentos específicos, mas nem por isso, estanques, uma vez que se articulam e se complementam. Este modelo foi apontado como fortaleza durante a avaliação, pois possibilitou o entendimento e a clareza do que deve ser feito, como deve ser feito e qual é o papel de cada um neste processo.

Percebe-se assim que não é possível olhar para um indivíduo, sem enxergar sua família e esta, dentro de uma comunidade, que vive em um determinado território. E uma vez feito isto, é necessário que o estudante verifique como o serviço de saúde se organiza considerando a realidade desta comunidade, família e pessoa e como se articulam as diferentes profissões frente as necessidade de saúde da população. Por isso, o paradigma da educação interprofissional escolhido busca alcançar esta imagem objetivo que está em consonância com o mercado de trabalho, sobretudo àquele referenciado pelo Sistema Único de Saúde.

Alguns estudos têm demonstrado que, dentre as diferentes categorias profissionais, predomina a tendência em satisfazer suas aspirações e manter sua autonomia, em detrimento da colaboração profissional (D'AMOUR, 1997). Tal postura espelha a própria constituição das profissões, que possuem em comum os interesses pragmáticos de garantia de mercado de trabalho. Conforme nos lembram estudos realizados pela sociologia das profissões, o profissionalismo constitui-se na história como estratégia de retenção do conhecimento, buscando torná-lo o mais específico e misterioso possível, permanecendo acessível a poucos e assim, garantindo reserva de mercado (MACHADO, 1995).

A educação Interprofissional pode ser conceituada como uma proposta onde duas ou mais profissões aprendem juntas sobre o trabalho conjunto e sobre as especificidades de cada uma, na melhoria da qualidade no cuidado ao paciente. (UNIFESP, 2009). É, considerada um estilo de educação que prioriza o 
trabalho em equipe, a integração e a flexibilidade da força de trabalho que deve ser alcançada com um amplo reconhecimento e respeito às especificidades de cada profissão. Isto pôde ser observado nas falas de professores e estudantes e, apesar de ainda haver um desconhecimento tanto de professores como de estudantes acerca do conceito de educação interprofissional, também parece haver consenso que o trabalho em equipe e a melhoria do atendimento aos pacientes estão presentes nas propostas pedagógicas.

Assim, a inserção responsável e comprometida de estudantes e docentes nos cenários reais, desde a primeira série dos cursos, visa à integração teórico/ prática, ensino/serviço e educação interprofissional sendo o alicerce em torno do qual se tece uma nova forma de ser, fazer, conhecer e conviver. O trabalho em equipe apontado como sendo uma fortaleza na atenção primária à saúde possibilita que os estudantes vivenciem o contexto da prática para: a) poderem exercitar a iniciativa; b) serem capazes de realizar análises "para identificar, planejar e propor soluções a problemas"; c) investirem em trabalho em equipe, abarcando a integralidade do cuidado na lógica da vigilância à saúde. Segundo Gyamarti (1986) a educação interprofissional possibilita integrar cuidado especializado com cuidado holístico, opondo-se ao reducionismo e a fragmentação da visão especializada.

Para que isso ocorra é necessário mais do que reunir estudantes de cursos diferentes em atividades conjuntas. Devem ser desenvolvidos recursos cognitivos, afetivos e psicomotores dos estudantes, tendo em mente que a identificação e o atendimento das necessidades de saúde das pessoas em questão, nas situações apresentadas, respeitem o grau de autonomia e domínio de cada profissão. Sendo assim, torna-se possível entender como as múltiplas dimensões de qualquer problema de saúde e a sua abordagem por distintas profissões se apresentam e se articulam na determinação do processo saúde-doença.

As peculiaridades do setor saúde o tornam especialmente interessante no estudo da formação profissional, uma vez que apresenta grande especialização em categorias e procedimentos e, ao mesmo tempo, vem sendo pressionado para diminuir a fragmentação de suas abordagens aos pacientes, seja por razões de eficácia ou mesmo de equacionamento de custos. Trata-se ainda de um setor no qual prevalecem profissionais liberais de várias formações que obedecem (e frequentemente privilegiam) orientações exteriores à organização, como aquelas provenientes dos conselhos de classe. Além disso, os membros das equipes de saúde atuam sobre problemas de extrema complexidade, realizando tarefas interdependentes e sobre as quais possuem controle relativo e cujos desdobramentos são sempre incertos, já que condicionados por vários fatores (D'AMOUR, 1997).

Conforme discutido por Almeida Filho (1997), a interdisciplinaridade ou 
a formação interprofissional não se efetiva através de princípios ou de intenções genéricas desenvolvidas em textos de pesquisadores bem intencionados. A troca efetiva e colaboração entre disciplinas e profissões serão possíveis somente pela ação de agentes concretos que irão ou não consubstanciar práticas mais ou menos integradas. Como ilustrado pelo autor, é somente através da concretude dos aparelhos cognitivos de indivíduos que transitem em diferentes áreas que ocorrerão os diferentes graus de interação e colaboração entre disciplinas e profissões (ALMEIDA FILHO, 1997).

Segundo Carpenter (1995), a convivência e a educação interprofissional, modificam atitudes e percepções negativas entre os profissionais de saúde remediando falhas na confiança e comunicação. Freeth (2002) também aponta que esta estratégia propicia maior conscientização e preparo para a prática, contribuindo para a socialização entre os profissionais de saúde. Cooper et al (2005) avaliando a educação interprofissional com estudantes de medicina, enfermagem, fisioterapia e terapia ocupacional na Universidade de Liverpool constatou o desenvolvimento de maior confiança e reforço da identidade profissional.

Portanto, estudantes de diferentes cursos, ao levarem em conta as necessidades de saúde individuais e coletivas para propor medidas que ampliem o cuidado e, consequentemente, que melhorem a qualidade de vida das pessoas, aprendem a aprender juntos sobre si, sobre o outro e sobre as diferentes profissões. Isto foi verificado por McNair et al (2005) que observaram a ampliação das experiências no trabalho de equipe na prática interprofissional com estudantes de medicina, farmácia e fisioterapia.

$\mathrm{Na}$ Famema, à medida que o curso avança as discussões mantém-se deflagradas pelos casos mais prevalentes, nas áreas de abrangência das Unidades de Saúde da Família, ampliando-se o levantamento das necessidades de saúde, discutindo-se causas e riscos, cuidando-se especialmente de integrar células, órgãos, sistemas, pessoas, desejos, vontades, família, condições sócioculturais e econômicas e serviços de saúde. Casto e Júlia (1994) estudando educação interprofissional e a prática colaborativa, apontaram que os profissionais desenvolvem a capacidade de lidar com problemas que excedem a capacidade de uma profissão isoladamente. Isto fortalece o compartilhamento de competências colaborativas porque inverte a lógica da formação tradicional em saúde, possibilitando que as profissões aprendam juntas sobre o trabalho conjunto e sobre as especificidades de cada uma na melhoria da qualidade do cuidado ao paciente. (BARR, 1998).

Entretanto, uma questão importante parece preocupar tanto professores como estudantes, e refere-se ao conteúdo e a aquisição de conhecimento cognitivo ao longo do processo. 
Os resquícios da educação baseada em conteúdos continuam a assombrar escolas, educadores e estudantes mesmo considerando que a quantidade de conhecimento científico produzido atualmente não pode ser transmitida em sua totalidade. Não podemos nos esquecer, da retórica de muitos professores que acreditam que a aquisição de conhecimento ocorre a partir dos conhecimentos "básicos" previamente aprendidos. Ora, se falamos de um modelo educacional que privilegia a aprendizagem a partir da prática profissional e do desenvolvimento da capacidade de aprender a aprender, os conteúdos que angustiam os professores e estudantes deveriam ser encarados como secundários.

Abrahamson (1978) já apontava o excesso de conteúdos comosendouma das principais "doenças curriculares". Isto a que ele chamou de "hipertrofia curricular" ainda está presente em muitas escolas e impedem que modificações curriculares sejam implementadas, pois exige cada vez mais tempo para a introdução de cada vez mais conteúdos de cada especialidade.

Portanto, acreditar que é possível ensinar tudo a todos e que o professor é quem continua a determinar conteúdos e complexidades a serem ensinadas parece se contrapor com o atual momento da educação mundial. Segundo Paulo Freire (1996, p.54), "O educador já não é aquele que apenas educa, mas o que, enquanto educa, é educado, em diálogo com o educando, que ao ser educado, também educa...".

Finalmente, mas não menos importante, a avaliação que deve ser considerada como um importante pilar para a efetividade desta proposta ganhando uma dimensão integradora dos aspectos cognitivos, afetivos e psicomotores. Assim, é necessário transcender a visão restrita e limitada da avaliação baseada apenas no quantitativo, para uma visão mais ampla onde o qualitativo se apresenta de forma complementar. Segundo Demo,

É um equívoco pretender confronto dicotômico entre qualidade e quantidade pela simples razão de que ambas as dimensões fazem parte da realidade da vida. Não são coisas estanques, mas facetas do mesmo todo. Por mais que possamos admitir qualidade como algo mais e mesmo melhor que quantidade, no fundo uma jamais substitui a outra, embora seja possível preferir uma à outra (DEMO, 1998, p. 89).

Dentro desta perspectiva formativa, Sousa (1995), Hoffmann (2001) e Silva (2006) acrescentam ainda a função energizante, motivadora, promotora como foi constato nos relatos de professores e estudantes. Segundo os autores o aluno sente-se estimulado ao perceber uma finalidade na avaliação que o professor 
propõe. E o professor passa a ter a tarefa de encantar os estudantes para que se tornem seres conscientes de seu papel enquanto aprendiz e indivíduo com responsabilidade social.

Entretanto, para que a avaliação torne-se de fato um momento de aprendizado e não de acerto de contas entre professor e estudante, como relatado por alguns durante a avaliação, a revisão do papel do professor como quem confere conceitos ou certificações de aprovação ou desaprovação devem ser revistos. Romão argumenta que:

Ou seja, realizada a avaliação da aprendizagem com o aluno, os resultados não devem constituir uma monografia ou uma dissertação do professor sobre os avanços e recuos do aluno, nem muito menos uma preleção corretiva dos erros cometidos, mas uma reflexão problematizada coletiva a ser desenvolvida ao aluno, para que ele, com o professor, retomem o processo de aprendizagem. Neste sentido, a sala de aula se transforma em um verdadeiro círculo de investigação do conhecimento e dos processos de abordagem do conhecimento (ROMÃO, 2003, p. 102)

Assim a avaliação passa a ter a função, de por si só, constituir-se em atividade de conhecimento e um ato de aprendizagem, assegurando o objetivo principal da educação interprofissional que é o de garantir o progresso formativo e educativo tanto de quem ensina como de quem aprende.

\section{Conclusões}

Ao fazermos a análise dos aspectos avaliativos apontados por professores e estudantes concluímos que a Famema está avançando na proposta da educação interprofissional na graduação para formar profissionais mais preparados para o trabalho em equipe e integralidade da atenção à saúde. Entretanto, algumas fragilidades apontadas demonstram que existem desafios a serem vencidos e referem-se à articulação do projeto com os serviços de saúde tornando o conceito de educação interprofissional conhecido por todos; aproximação dos estudantes e profissionais de saúde melhorando o trabalho em equipe; rompimento com o paradigma do ensino tradicional centrado em conteúdos e o aprimoramento das estratégias de avaliação. 


\section{REFERÊNCIAS}

ABRAHAMSON, S. J. Diseases of Curriculum. Medical Education, v. 53, n. 12, p. 951-957, 1978.

ALMEIDA FILHO, N. Transdisciplinaridade e Saúde Coletiva. Ciência \& Saúde Coletiva, 2(1/2), p. 5-20, 1997.

BARR, H. Competent to collaborate; towards a competency-based model for interprofessional education. Journal of Interprofessional Care, v. 12, n. 2, p. 181-188, 1998.

BATISTA, N. A.; BATISTA, S. H. S. S. A prática como eixo da aprendizagem na graduação médica. In: PUCCINI, R. F. et al. (Org.). A formação médica na Unifesp: excelência e compromisso social. São Paulo: Editora Unifesp, 2008. 312p.

BRASIL. Ministério da Saúde. Secretaria de Recursos Humanos. I Conferência Nacional de Recursos Humanos para a Saúde: relatório final. Cadernos RH Saúde, Brasília, v. 1, n. 1, p. 141-184, nov. 1993.

CARPENTER, J. Interprofessional education for medical and nursing students: evaluation of a programme. Medical Education, 29, p. 265-272, 1995.

CASTO, R. M.; JULIA, M. C. Interprofessional care and collaborative practice. Pacific Grove: Brooks/Cole Publishing Company, 1994.

CONTRANDIOPOULOS, A. P. et al. A avaliação na área da saúde: conceitos e métodos. In: HARTZ, Z. M. A. (Org.). Avaliação em saúde: dos modelos conceituais a prática na análise da implantação de programas. Rio de Janeiro: Edit. FIOCRUZ, 1997. p. 29-47,

COOPER, H.; SPENCER-DAWE, E.; MCLEAN, E. Beginning the process of teamwork: design, implementation and evaluation of an inter-professional education intervention for first year undergraduate students. Journal of Interprofessional Care, v. 19, n. 5, p. 492-508, 2005.

D'AMOUR, D. Structuration de la collaboration interprofessionelle dans les services de santé de première ligne au Québec. Tese (Doutorado) - Université de Montreal, Montreal/Canadá, 1997.

DELORS, J. (Org.). Educação: um tesouro a descobrir: relatório para a UNESCO da Comissão Internacional sobre Educação para o Século XXI. 4. ed. São Paulo: Cortez, 2000.

DEMO, P. Pesquisa qualitativa. Busca de equilíbrio entre forma e conteúdo. Ver. LatinoAm. Enfermagem, Ribeirão Preto, v. 6, n. 2, p. 89-104, abril 1998. 
FREETH, D. et al. A critical review of interprofessional education. 2002. Disponível em: <www.caipe.org.uk/publications $>$. Acesso em: 05/07/2006.

FREIRE, P. Pedagogia da autonomia. Saberes necessários à prática educativa. São Paulo: Paz e Terra, 1996. p. 54.

GOELEN, G. et al. Measuring the effect of interprofessional problem-based learning on the attitudes of undergraduate health care students. Medical Education, v. 40, n.6, p. 555-561, 2006.

GOMES, R. et al. Organização, processamento, análise e interpretação dos dados: o desafio da triangulação. In: MINAYO, M. C. S. et al. (Org.). Avaliação por triangulação de métodos: abordagem de programas sociais. Rio de Janeiro: Fio Cruz, 2005. cap 6, p. $185-221$.

GYAMARTI, G. The teaching of the professions: an interdisciplinary approach. Higher Education Review, v. 18, n.2, p. 33-43, 1986.

HIND, M. et al. Interprofessional perceptions of health care students. Journal of Interprofessional Care,;v. 17, n.1, p. 21-34, Feb. 2003.

HOFFMANN, J. Avaliar para promover: as setas do caminho. Porto Alegre. Ed. Meditação, 2001.

MACHADO, M. H. Sociologia das profissões: uma contribuição ao debate teórico. In: MACHADO, M. H. (Org.). Profissões de saúde: uma abordagem sociológica. Rio de Janeiro: Fiocruz, 1995.

MCNAIR, R.; STONE, N.; SIMS, J.; CURTIS, C. Australian evidence for interprofessional education contributing to effective teamwork preparation and interest in rural practice. Journal of Interprofessional Care, 19, p. 579-594, 2005.

MINAYO, M. C. S. Os efeitos da globalização no mundo do trabalho e políticas públicas. In: SEMINÁRIO NACIONAL DE DIRETRIZES PARA A EDUCAÇÃO EM ENFERMAGEM NO BRASIL, 2., 1997. Florianópolis: ABEn-SC, p. 35-44, 1997.

MINAYO, M. C. S. O desafio do conhecimento. São Paulo: Hucitec, 2003.

MINAYO, M. C. S. et al. Métodos, técnicas e relações em triangulação. In: MINAYO, M. C. et al. (Org.). Avaliação por triangulação de métodos: abordagem de programas sociais. Rio de Janeiro: Editora Fiocruz, 2005.

MINAYO M. C. S.; SANCHES, O. Qualitativo-quantitativo: oposição ou complementaridade? Cadernos de Saúde Pública, Rio de Janeiro, v. 9, n. 3, p. 239248, jul./set. 1993. 
MOTTA, J. I. J.; BUSS, P.; NUNES, T. C. M. Novos desafios educacionais para a formação de recursos humanos em saúde. Olho Mágico, Londrina, v. 8, n. 3, p. 4-8, set./dez. 2001.

NOGUEIRA, R. P. (Coord.). Avaliação das tendências e prioridades sobre recursos humanos de saúde. Brasília: Organização Pan-Americana da Saúde, 2002.

ROMÃO, J. E. Avaliação dialógica desafios e perspectivas. 5. ed. São Paulo: Cortez: Instituto Paulo Freire, 2003

SANTOS, B. S. Pela mão de Alice: o social e o político na pós-modernidade. 6. ed. São Paulo: Cortez, 1999.

SILVA, J. F. Avaliação na perspectiva formativa-reguladora. Pressupostos teóricos e práticos. 2. ed. Porto Alegre: Meditação, 2006.

SOUSA, C. Descrição de uma trajetória na/da Avaliação educacional. Ideias, São Paulo, FDE, v. 30, p. 161-174, 1995.

TSUJI, H.; SILVA, R. H. A. Faculdade de Medicina de Marília e sua relação com a medicina de família e comunidade. Revista Brasileira de Medicina de Família e Comunidade, v. 01, p. 26-33, 2006.

UNIFESP (2009) O Projeto Pedagógico do Campus Baixada Santista. Disponível em: $<$ http://www.baixadasantista.unifesp.br/projpedag.php $>$. Acesso em: 06/07/2009.

Texto recebido em 27 de maio de 2009

Texto aprovado em 26 de agosto de 2009 